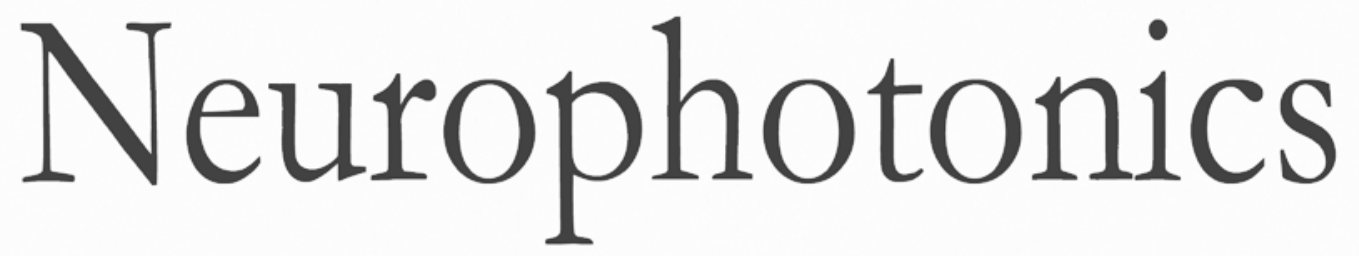

\title{
Computer-generated holography enhances voltage dye fluorescence discrimination in adjacent neuronal structures
}

\author{
Amanda J. Foust \\ Valeria Zampini \\ Dimitrii Tanese \\ Eirini Papagiakoumou \\ Valentina Emiliani
}




\title{
Computer-generated holography enhances voltage dye fluorescence discrimination in adjacent neuronal structures
}

\author{
Amanda J. Foust, ${ }^{a}$ Valeria Zampini, ${ }^{a}$ Dimitrii Tanese, ${ }^{a}$ Eirini Papagiakoumou, ${ }^{a, b}$ and Valentina Emiliani ${ }^{a, *}$ \\ ${ }^{a}$ Paris Descartes University, Biomedical and Fundamental Science Faculty, Wavefront-Engineering Microscopy Group, Neurophotonics \\ Laboratory, CNRS UMR8250, 45, rue des Saints Pères, 75270 Paris Cedex 06, France \\ bINSERM, 45, rue des Saints Pères, 75270 Paris Cedex 06, France
}

\begin{abstract}
Voltage-sensitive fluorescence indicators enable tracking neuronal electrical signals simultaneously in multiple neurons or neuronal subcompartments difficult to access with patch electrodes. However, efficient widefield epifluorescence detection of rapid voltage fluorescence transients necessitates that imaged cells and structures lie sufficiently far from other labeled structures to avoid contamination from out of focal plane and scattered light. We overcame this limitation by exciting dye fluorescence with one-photon computer-generated holography shapes contoured to axons or dendrites of interest, enabling widefield detection of voltage fluorescence with high spatial specificity. By shaping light onto neighboring axons and dendrites, we observed that dendritic back-propagating action potentials were broader and slowly rising compared with axonal action potentials, differences not measured in the same structures illuminated with a large "pseudowidefield" (pWF) spot of the same excitation density. Shaped illumination trials showed reduced baseline fluorescence, higher baseline noise, and fractional fluorescence transient amplitudes two times greater than trials acquired with pWF illumination of the same regions. ๑ The Authors. Published by SPIE under a Creative Commons Attribution 3.0 Unported License. Distribution or reproduction of this work in whole or in part requires full attribution of the original publication, including its DOI. [DOI: 10.1117/1.NPh.2.2.021007]
\end{abstract}

Keywords: computer-generated holography; voltage imaging; action potential; axon; dendrite.

Paper 14071SSR received Oct. 9, 2014; accepted for publication Dec. 5, 2014; published online Jan. 7, 2015.

\section{Introduction}

Understanding neuronal input-output transformations requires experimental characterization of how electrical signals generate and propagate in axonal and dendritic arbors. Although whole cell patch clamp techniques have enabled high temporal resolution and high signal-to-noise ratio $(\mathrm{S} / \mathrm{N})$ electrical recordings in somata and large dendrites, smaller structures remain difficult to access with patch electrodes. Voltage-sensitive dyes (VSDs) provide an alternative method to track membrane potential and have been effectively imaged with one-photon epifluorescence microscopy to characterize action potential propagation in small diameter axons and dendrites. ${ }^{1-6}$ Due to the close spatial mingling of neuronal substructures, improving lateral and axial confinement with confocal microscopy could enable discrimination of signals arising from adjacent or overlapping structures; however, the relatively low fractional sensitivity of voltage sensors (dF/F 5\% to $20 \%$ per $100 \mathrm{mV}$ in brain slices) necessitates excitation densities and collection efficiencies sufficient to overcome high fractional shot noise. ${ }^{7-9}$ Loss of photon flux through confocal pinhole and lens arrays stipulates extensive signal averaging ${ }^{10}$ or long integration times ${ }^{11}$ to increase the S/N. Among efforts to measure voltage transients with two-photon fluorescence, ${ }^{12,13}$ Acker et al. ${ }^{14}$ achieved high spatial specificity by implementing two-photon excitation in "single voxel" (nonscanning) mode to record voltage transients in single dendritic spines. However, low fractional voltage dye sensitivity, combined with

*Address all correspondence to: Valentina Emiliani, E-mail: valentina.emiliani@ parisdescartes.fr fast sampling rates necessitated by rapid voltage transients, limits the number of points from which high $\mathrm{S} / \mathrm{N}$ voltage-sensitive fluorescence changes can be collected in serial.

Here we propose an alternative method to obtain voltage signals from specific structures of interest while preserving the ability to record from several spatial locations in parallel. To this end, we implemented one-photon computer-generated holography $(\mathrm{CGH})^{15}$ to target VSD excitation over laterally extended regions, imaging epifluorescence signals in widefield mode with a high frame-rate camera. Cells filled with voltage dye were imaged and based on structure shape and location, a phase profile was calculated and addressed to a spatial light modulator (SLM) such that illumination at the sample plane was confined to the dendrite or axon of interest. In contrast with full-field illumination, patterned excitation minimized signal degradation arising from nonspecific autofluorescence, spilled dye, or nearby labeled structures. Importantly, with CGH-shaped illumination we could discriminate kinetic differences between action potential-evoked fluorescence transients recorded from neighboring axons and dendrites, differences that could not be appreciated with widefield illumination. These results suggest that $\mathrm{CGH}$ can provide a scanless means to increase the spatial specificity of functional fluorescence measurements.

\section{Methods}

\subsection{Slice Preparation, Cell Loading, and Recording}

We prepared slices of somatosensory cortex from wild-type mice $(\mathrm{C} 57 \mathrm{BL} / 6 \mathrm{~J})$ in accordance with the guidelines of European Union and institutional guidelines of the care and use of 
laboratory animals (Council directive 86/609 European Economic Community). Male or female mice (3 to 4 weeks of age) were deeply anesthetized with isoflurane (5\%) and killed through decapitation. The brain was rapidly removed and placed in ice-cold $\left(<5^{\circ} \mathrm{C}\right)$ or room temperature cutting solution containing the following (in $\mathrm{mM}$ ): 110 choline chloride, $2.5 \mathrm{KCl}, 7.0$ $\mathrm{MgCl}_{2}, 0.5 \mathrm{CaCl}_{2}, 25 \mathrm{NaHCO}_{3}, 1.25 \mathrm{NaH}_{2} \mathrm{PO}_{4}, 20$ glucose, and aerated with $95 \% \mathrm{O}_{2}, 5 \% \mathrm{CO}_{2}$ to a final $\mathrm{pH}$ of 7.4. We dissected the cerebral cortex, blocking it to take coronal slices of somatosensory cortex. The cortical block was glued to an icecold stage on a Leica microslicer, and $300 \mu \mathrm{m}$ slices were cut in ice-cold cutting solution. Cut sections were placed in an incubator at $35^{\circ} \mathrm{C}$ for $0.5 \mathrm{~h}$ in the cutting solution in a chamber containing ACSF (in $\mathrm{mM}$ ): $125 \mathrm{NaCl}, 2.5 \mathrm{KCl}, 1.0 \mathrm{MgCl}_{2}$, $2.0 \mathrm{CaCl}_{2}, 25 \mathrm{NaHCO}_{3}, 1.25 \mathrm{NaH}_{2} \mathrm{PO}_{4}, 20$ glucose, and 0.4 L-ascorbic acid. Slices were kept at room temperature for at least $0.5 \mathrm{~h}$ before dye loading and recording in a submersion chamber.

\subsection{Dye Loading}

We visualized recovered cortical slices with Olympus BX51WI's native infrared differential-interference contrast (IR-DIC) path, further magnifying the image onto a scientific complementary metal oxide semiconductor (sCMOS, ORCA Flash 4, Hamamatsu) camera with a $2 \times / 4 \times$ magnification changer (Fig. 1 "MC"; Luigs and Neumann, Ratingen, Germany) mounted above the tube lens. We loaded neurons with VSD with a glass patch electrode by filling the tip with dye-free internal solution (in mM: $130 \mathrm{~K}$-gluconate, $7 \mathrm{KCl}, 4$ ATP-Mg, 0.3 GTP-Na, 10 phosphocreatine-Na, 10 HEPES; adjusted to $\mathrm{pH} 7.4$ and $284 \mathrm{~mol} / \mathrm{kg}$ ), then back-filling with the dye-containing internal solution (JPW3028, 400 to $800 \mu \mathrm{M}$ ), and performed whole-cell somatic recordings from the selected neuron under DIC optics. The dye JPW3028 is a doubly positively charged analog of the aminostyryl-pyridinium series of lipophilic VSDs available from Invitrogen as D-6923. This electrochromic dye does not significantly increase the membrane capacitance of the labeled neuron, as evident from several control measurements showing that the waveform of the electrically recorded action potentials remains unaltered after intracellular dye loading. ${ }^{16}$ Dye-free solution in the tip is necessary because this highly lipophilic molecule would otherwise spill onto the slice before sealing and patching the cell of interest, as any dye bound to membrane outside the cell of interest increases the background fluorescence and thus degrades recording sensitivity. The cell was patched and stained at room temperature for 15 to $30 \mathrm{~min}$ by allowing passive diffusion of the dye into the cell. The dye diffuses slowly compared with other dyes of the same size and weight due to its high affinity for lipid membranes. After staining, we carefully detached the patch electrode from the neuron, forming an outside-out patch, after which the slice was incubated at room temperature for 1 to $2 \mathrm{~h}$, allowing the dye to diffuse from the soma into the axon and neighboring basal dendrites.

\subsection{Computer-Generated Holography}

Dye-loaded cells were illuminated with a $450 \mathrm{~mW}$ frequencydoubled diode-pumped $\mathrm{Nd}: \mathrm{YVO}_{4}$ low-noise laser emitting at $532 \mathrm{~nm}$ (Fig. 1 "LS"; MLL-FN-532-450-5-LAB-TTL, Changchun New Industries Optoelectronics Tech. Co. Ltd., Changchun, China) with CGH to achieve desired spatial patterns of light at the objective focal plane. Specifically, the laser beam (Fig. 1, LS) is attenuated with a neutral density (ND) filter, a half-wave plate $(\lambda / 2)$ and polarizing beam splitter, reading and adjusting the power with a removable mirror (M2), and a power meter (PM, Newport 818-ST2). Lenses $\mathrm{L}_{\mathrm{BE} 1}$ $(f=19 \mathrm{~mm})$ and $\mathrm{L}_{\mathrm{BE} 2}(f=150 \mathrm{~mm})$, and pinhole $(\mathrm{PH}$, $d=15 \mu \mathrm{m}$ ) clean and expand the beam to fill the active area of the liquid crystal on silicon spatial light modulator (LCOS-SLM, Hamamatsu X10468-01). Lens L $\mathrm{L}_{1}(f=400 \mathrm{~mm})$ converts the modulated wavefront into a spatial light pattern, which is demagnified into the sample plane by a telescope formed by $\mathrm{L}_{1}, \mathrm{~L}_{2}(f=200 \mathrm{~mm})$ and the microscope objective (Fig. 1 "OBJ," Olympus LUMFLN 60XW, NA = 1.1, $f=5 \mathrm{~mm})$. The 532-nm fluorescence excitation light is reflected through the objective by a 560-nm long-pass dichroic (FF560-FDi01-25x36, Semrock, Rochester, New York). Fluorescence transmitted by the dichroic is long-pass filtered ( $\mathrm{F}_{\mathrm{EM}}$, 593LP, FF01-593/LP-25, Semrock) and imaged onto a high speed scientific-CMOS camera (sCMOS, Hamamatsu ORCA Flash 4.0) or electron multiplying charge-coupled device (EMCCD, Andor 860 iXon3) by the native Olympus tube lens $\left(\mathrm{L}_{\mathrm{TB}}\right)$ and $\mathrm{MC}$ used to target patch clamp electrodes. In order to block the zero-order non phase-modulated component reflected from the SLM, we introduced a defocus in the beam by adjusting the distance between $\mathrm{L}_{\mathrm{BE} 1}$ and $\mathrm{L}_{\mathrm{BE} 2}$ in order to displace the zero-order focus by 30 to $40 \mathrm{~mm}$ after the Fourier plane of the first lens. ${ }^{17,18}$ For the diffracted first order, the defocus was compensated with a spherical Fresnel lens at the SLM. Thus, with the zero-order displaced 30 to $40 \mathrm{~mm}$ from the effective Fourier plane of $\mathrm{L}_{1}$, we could block the unwanted zero-order component with a point block [Fig. 1(a), "B1 0 " tape on cover slip] without perturbing the propagation of the hologram (first-order beam). Phase holograms were calculated with an iterative Fourier transform algorithm (IFTA). ${ }^{19-22}$ The IFTA-generated phase profiles were computed and addressed to the SLM using "Wavefront Designer IV," in house software written in C++ with Qt 4.4.0 and fftw 3.1.2. ${ }^{23}$

After the dye diffusion period, we imaged the neuron's dendrites and axon at depths of 10 to $50 \mu \mathrm{m}$, weakly illuminating (excitation density $\sim 1 \mathrm{nW} / \mu \mathrm{m}^{2}$ ) with a CGH-generated square shape $(100 \mu \mathrm{m})$ leaving a light-free slot in the center $\sim 30 \mu \mathrm{m}$ wide [see Fig. 1(b)]. Positioning the soma in this light free slot enabled examination of axonal and dendritic structures, while sparing the dye-filled soma from unnecessary photodamage. Low-power density illumination was paired with long (500 ms) camera integration time and binning $(2 \times 2$ or $4 \times 4)$ to maximize collection and minimize light exposure and photodamage to stained axons and dendrites during preimage acquisition. We then used fluorescence images of the axon and dendrites to define the spatial patterns of illumination for VSD signal acquisition trials, sculpted to the structure's contours [Fig. 1(c)]. For VSD signal acquisition, we decreased the frame integration time to $156 \mu$ s (frame rate $=6.41 \mathrm{kHz}$ ) and modulated the laser power to achieve an excitation density in the range of 0.6 to $10 \mu \mathrm{W} / \mu \mathrm{m}^{2}$ across the axon- or dendriteshaped region. Laser light was gated onto the cell with a highspeed shutter [Fig. 1(a)] "Sh"; Uniblitz LS6, driver D880C; Rochester, New York] for 10 to $30 \mathrm{~ms}$ trials, with 1 to $2 \mathrm{~min}$ intertrial intervals. During each trial, we stimulated action potentials with brief current pulses ( 5 to $10 \mathrm{~ms}, 400$ to $800 \mathrm{pA}$ ) applied through a dye-free pipette patched in whole-cell configuration. Electrical and optical waveforms were monitored 
(a)

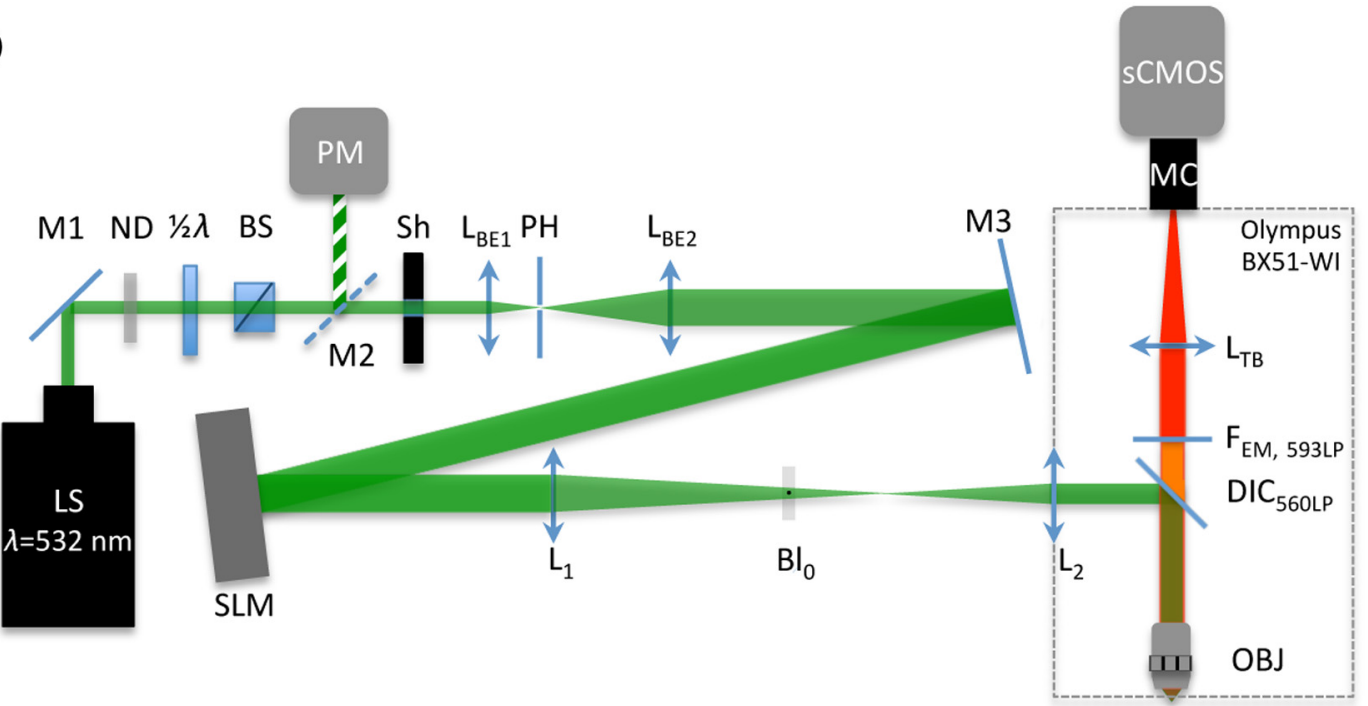

(b)

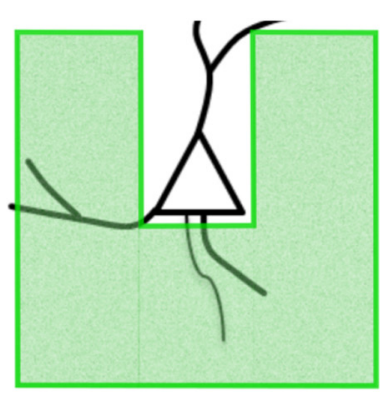

(c)

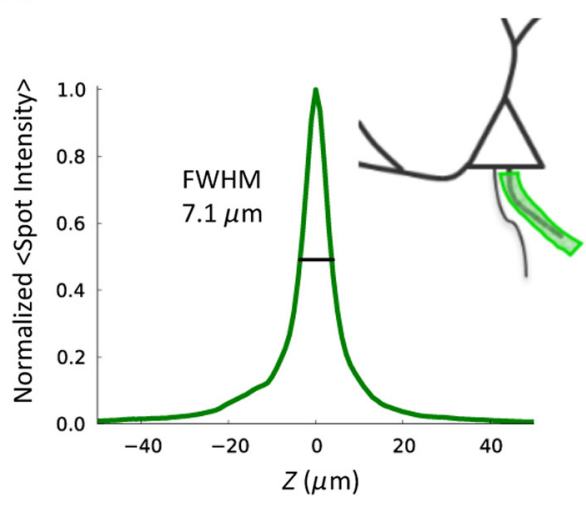

(d) Pseudo wide-field axial profile

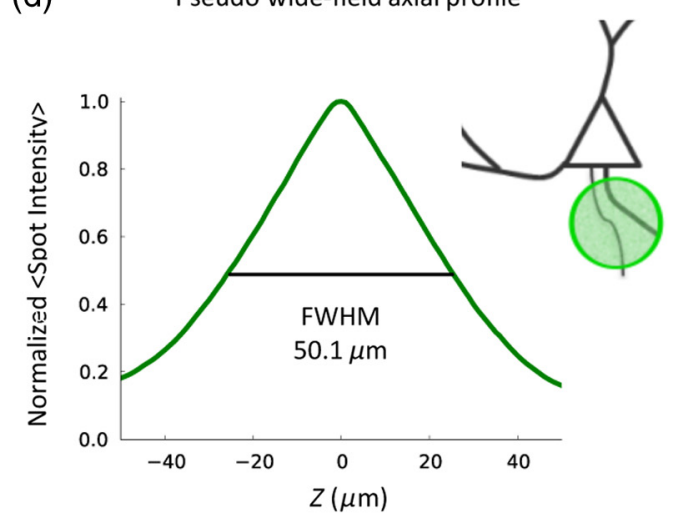

Fig. 1 Computer-generated holography (CGH) schematic and axial propagation. (a) The beam emitting from the solid-state $532 \mathrm{~nm}, 450 \mathrm{~mW}$ laser (LS) is attenuated with a neutral density filter (ND), a half-wave plate $(\lambda / 2)$ and polarizing beam splitter (BS), reading and adjusting the power with removable mirror (M2), and a power meter $(\mathrm{PM})$. Lenses $\mathrm{L}_{\mathrm{BE} 1}(f=19 \mathrm{~mm})$ and $\mathrm{L}_{\mathrm{BE} 2}(f=150 \mathrm{~mm})$, and pinhole $(\mathrm{PH}, d=15 \mu \mathrm{m})$ clean and expand the beam to fill the active area of the spatial light modulator. Lens $L_{1}(f=400 \mathrm{~mm})$ converts the modulated wavefront into a spatial light pattern, which is demagnified into the sample plane by a telescope formed by $\mathrm{L}_{2}(f=200 \mathrm{~mm})$ and the objective $(\mathrm{NA}=1.1, M=60 \times, f=5 \mathrm{~mm})$. The zeroorder component is blocked by $\mathrm{Bl}_{0}$ before the beam enters the Olympus BX51WI microscope. The 532$\mathrm{nm}$ fluorescence excitation light is reflected through the objective by a 560LP dichroic. Fluorescence transmitted by the dichroic is long-pass filtered $\left(\mathrm{F}_{\mathrm{EM}}, 593 \mathrm{LP}\right)$ and imaged onto a high-speed scientific-CMOS camera (sCMOS) by the native Olympus tube lens $\left(\mathrm{L}_{\mathrm{TB}}\right)$ and magnification changer (MC). (b) Scheme of large shaped "square with slot" illumination used for imaging dendrites and axons before voltage imaging measurement. The squared spot $(100 \mu \mathrm{m})$ has a light-free region in the center (of around $30 \mu \mathrm{m}$ width) to spare the dye filled soma from unnecessary illumination and photodamage. The lateral shape masks (green) are specified based on widefield epifluorescence images of voltage-sensitive dye (VSD) filled structures. (c) Simulated axial propagation profile of light shaped to the contours of the axons or dendrites of interest. Full width at half maximum (FWHM) $z$-confinement is $7.1 \mu \mathrm{m}$. (d) Simulated axial propagation profile of large-spot "pseudowidefield" (pWF) illumination. FWHM $z$-confinement is $50.1 \mu \mathrm{m}$.

for significant changes in width and fall-time indicative of photodamage, at which point the experiment was discontinued. CGH-sculpted light illumination trials were interleaved with "pseudowidefield" (pWF) trials illuminated with a large diameter (25 to $40 \mu \mathrm{m})$ spot with poor axial confinement [Fig. 1(d)], increasing laser power to maintain an excitation density equal to that of the sculpted light trials.

Current clamp signals were recorded at $20 \mathrm{kHz}$ with a MultiClamp 700B amplifier and 1440A digitizer (Molecular
Devices, Sunnyvale, California), which also triggered camera acquisition and shutter opening. Micromanager ${ }^{24}$ piloted the sCMOS and Andor Solis drove the EMCCD. We analyzed fluorescence signals during experiments with the Image ${ }^{25}$ time series plug-in and custom MATLAB scripts. Posthoc image visualization, processing, analysis, and statistics were performed in ImageJ and "VKAT: Voltage-imaging Kinetics Analysis Tool," a wxpython-based GUI leveraging modules Numpy and Matplotlib. 


\subsection{Axial Propagation Simulations}

In order to estimate and compare the $z$-confinement of $\mathrm{CGH}$ shaped and "pWF" configurations, we simulated the distribution of the holographic beam propagating along the optical axis around the objective focal plane as described in Ref. 26. Briefly, we calculated the beam irradiance around the objective focal plane after the input phase hologram propagates through the telescope formed by $\mathrm{L}_{1}, \mathrm{~L}_{2}$, and the objective using the angular spectrum approach of plane waves with a thin element approximation. Lutz et al. ${ }^{26}$ demonstrated that the simulations faithfully predict the experimentally measured propagation of shaped, CGH-generated spots. Although these simulations do not factor in depth-dependent brain tissue scattering, Zahid et al. ${ }^{18}$ have shown that holographic spots maintain axial confinement at depths of $30 \mu \mathrm{m}$ in hippocampal slices. The axial confinement of the simulated beam propagation was quantified as full width at half maximum (FWHM) of intensity averaged over the "dendrite shaped" region of interest (ROI) in each axial plane [Fig. 1(c)].

\subsection{Analysis}

Axons and dendrites were discriminated visually, axons being thin and straight with collaterals issuing at obtuse angles, and dendrites thicker, spiny, and branching off in acute angle "Y"s. We spatially averaged fluorescence signals from each imaged axon or dendrite over groups of 12 to 18 pixels. Acquiring frames at $6410 \mathrm{~Hz}$, our sCMOS camera operated in "rolling shutter" mode, resulting in a systematic exposure delay of each horizontal line on the chip. Any temporal distortion potentially induced by the rolling shutter was canceled by spatially averaging axon and dendrite ROIs over pixels located across similar numbers of horizontal lines. All data shown and reported come from single-trial measurements. We applied a low-pass binomial filter (1 to 2 passes) to reduce high-frequency noise. In order to improve the temporal precision of spike kinetic measurements, the data collected at 6410 frames/s were reconstructed using cubic spline interpolation, and then resampled to $100 \mathrm{kHz}$ before measuring spike width and rise time. Both for shaped and pWF trials, spike kinetic measurements were taken from ROIs in which both CGH excitation and widefield imaging were parfocal with the structure of interest. Axons and dendrites occupied different axial planes preventing simultaneous widefield epifluorescence imaging of both structures. Axonal and dendritic data were thus collected in separate, alternate trials. Because the $\mathrm{d} F / F$ signal from different parts of the neuron varies owing to factors other than voltage (e.g., partitioning of dye in inner and outer membranes), changes in voltage indicated by changes in fluorescence could not be calibrated on an absolute scale. We therefore limited comparison of signals emanating from axonal and dendritic compartments to kinetic parameters that do not depend on precise, absolute voltage scale calibration.

Since variable nonspecific fluorescence precludes signal amplitude comparisons between different ROIs, we performed paired comparisons of baseline fluorescence, noise, fractional change, and $\mathrm{S} / \mathrm{N}$ in compartments illuminated with shaped and large spot pWF in alternate trials. To quantify spike-evoked fluorescence amplitude, we calculated the fractional change in fluorescence $\mathrm{d} F$, with respect to the baseline fluorescence for each signal-bearing pixel. Specifically $\frac{\mathrm{d} F}{F}=\frac{F-F_{\mathrm{BL}}}{F_{\mathrm{BL}}-F_{\mathrm{dark}}}$,

where $F_{\text {dark }}$ is the average trace intensity $3 \mathrm{~ms}$ before the shutter opens and $F_{\mathrm{BL}}$ is the average "baseline" trace intensity during $3 \mathrm{~ms}$ after shutter opening but before spiking. For each trace, we also quantified the $\mathrm{S} / \mathrm{N}$ :

$\mathrm{S} / \mathrm{N}=\frac{\mu}{\sigma}$,

where

$\mu={\frac{\mathrm{d} F}{F_{\text {MAX }}}}$

and $\sigma=$ root-mean-square-deviation of samples acquired during "baseline" (BL, t = 1:n):

$\sigma=\sqrt{\frac{1}{n} \sum_{t=1}^{n}\left(\left.\frac{\mathrm{d} F}{F}\right|_{t}-\left\langle\frac{\mathrm{d} F}{F}\right\rangle_{\mathrm{BL}}\right)^{2}}$.

We quantified differences between the kinetics (spike width, rise time) and $\mathrm{S} / \mathrm{N}$ characteristics between axonal- and dendriticgenerated waveforms, as well as CGH and "pWF" (large spot) configurations.

\section{Results}

We utilized CGH to confine VSD fluorescence excitation light to an axon or dendrite of interest [Figs. 1(c), 2(b), and 2(c)], comparing signals obtained in this fashion to those illuminated in "pWF," that is with a large spot of light with poor axial confinement [Figs. 1(d) and 2(a)]. CGH-sculpted illumination generated an excitation spot that precisely covered the structure of interest and corresponded to approximately $1 / 20$ of the area of the 25 to $40 \mu \mathrm{m}$-diameter circles used for pWF trials [Figs. 1(c) and 1(d)]. Axial propagation simulations of $532 \mathrm{~nm}$ CGH-generated shapes predict axial confinement between 7.0 and $9.6 \mu \mathrm{m}$ for shaped illumination [Fig. 1(c)], and between 41 and $66 \mu \mathrm{m}$ for pWF spots [Fig. 1(d)]. Both CGH and "pWF" excitation of dye in axons and dendrites generated fluorescence signals from which spike waveforms could be resolved in single trials [Figs. 2(a)-2(c) and 2(e)].

\subsection{Targeted Fluorophore Excitation Enables Signal Discrimination in Neighboring Structures}

We found that CGH-shaped voltage dye fluorescence excitation in neighboring axons and dendrites enabled discrimination of differing action potential kinetics not possible with large-field illumination. Specifically, we compared $10 \%$ to $90 \%$ rise time and FWHM of action potential-evoked fluorescence transients recorded in axons and dendrites illuminated with CGHsculpted shapes in alternate trials (Fig. 2). Corresponding to previously reported electrical ${ }^{27,28}$ and optical measurements, ${ }^{2,4,5}$ action potential rise time and FWHM were shorter [ $p$-values $\leq$ 0.05 Student's $t$-test; Figs. 2(b), 2(c), 2(e), and 2(g)] in axons (FWHM: $1.62 \mathrm{~ms} \pm 0.24$ standard error of the mean, S.E.M.; rise time: $522.1 \mu \mathrm{s} \pm 95.6$ S.E.M., $n=3$ trials from two cells) than in dendrites (FWHM: $2.37 \mathrm{~ms} \pm 0.30$ S.E.M.; rise time: $1154.6 \mu \mathrm{s} \pm 194.0$ S.E.M.; $n=4$ trials from two cells). Displayed action potential-evoked fluorescence transients 
(a)

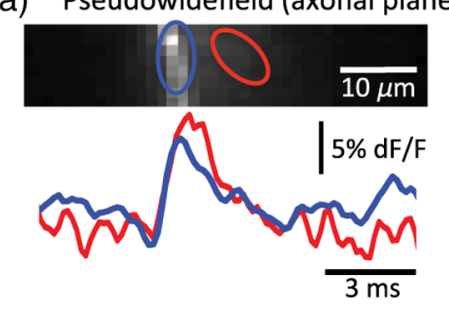

(b) CGH dendrite (dendritic plane)

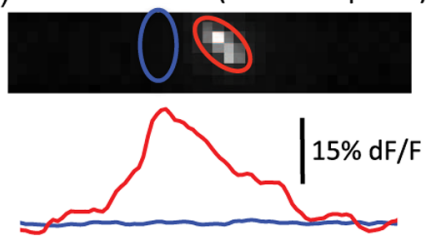

(c) CGH axon (axonal plane)

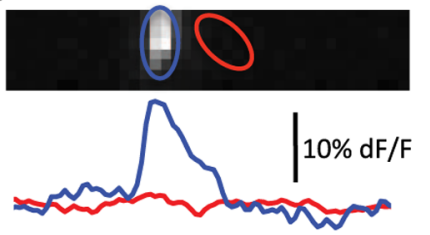

(d)
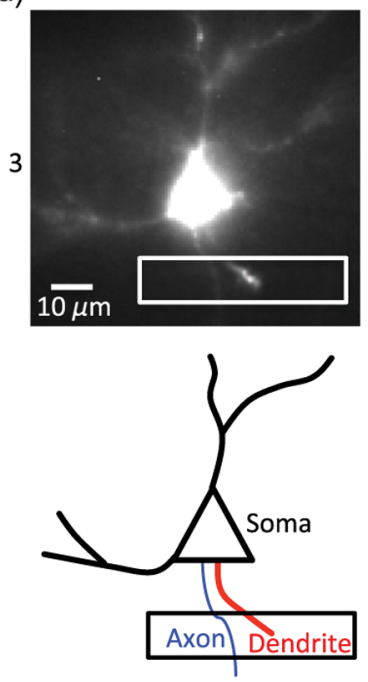

(e)

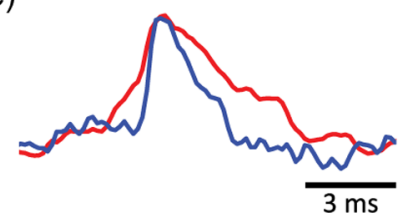

(f)

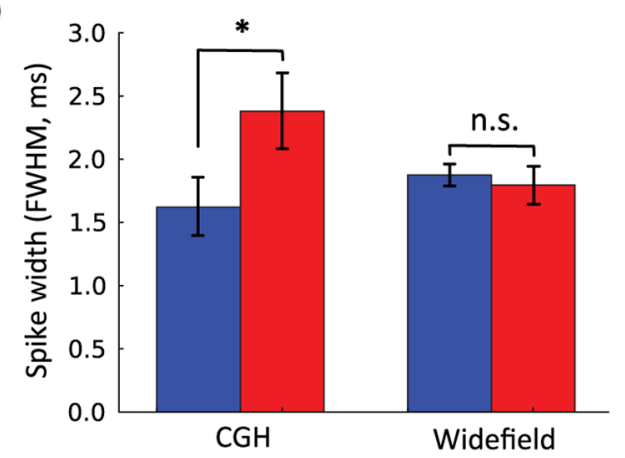

(g)

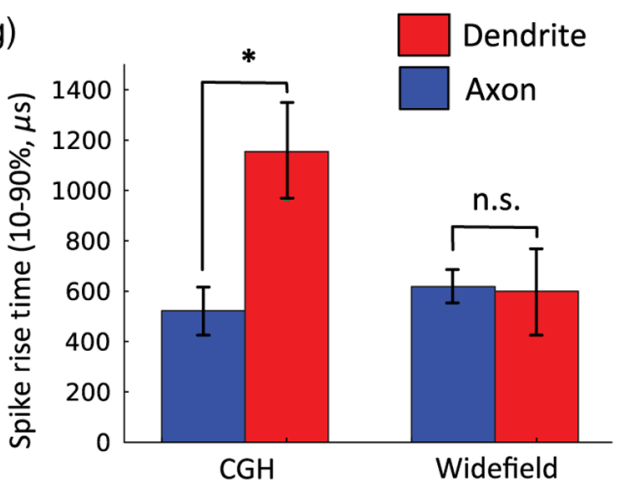

Fig. 2 Holographic light sculpting enables discrimination of spike kinetic properties from neighboring axons and dendrites. (a) VSD fluorescence transients from axonal (blue) and dendritic (red) regions of interest (ROIs) with large $(d=25 \mu \mathrm{m})$ spot "pWF" illumination. The axon (in focus in image) and dendrite (more superficial, out focus in image) occupy different axial planes. (b) VSD fluorescence transient emanating from a dendrite occupying a focal plane 5 to 10 microns deeper than the axon in panel (a). Light is holographically targeted to excite fluorescence only in dendritic tip ROI (red trace), without exciting fluorescence in the adjacent axon in an out-of-focus deeper axial plane (blue trace). (c) Light is holographically targeted to excite fluorescence only in axonal ROI (blue trace), without exciting fluorescence in the adjacent dendrite in an out-of-focus more superficial plane (red trace). Dendritic (panel b) and axonal (panel c) traces were acquired in separate trials. Traces in (a)-(c) and (e) represent single trials averaged over 12 to 18-pixel ROls collected at $6.41 \mathrm{kHz}$ and low pass filtered (filter type: binomial, 2 passes). (d) Fluorescence image and schematic of dye-filled pyramidal neuron and schematic (lower) showing position of recorded axon (blue) and dendrite (red). The image (upper) is a maximum intensity projection of fluorescence images collected in the deeper axonal plane and more superficial basal dendrite plane. (e) An overlay of axonal (blue) and dendritic (red) traces acquired in alternate trials (panels $\mathrm{b}$ and $\mathrm{c}$ ), peak-aligned and amplitude-normalized to show the faster rise-time and shorter duration of the axonal spike. (f) CGH-shaped excitation of dye in adjacent axons and dendrites (i.e., b and c), showed dendritic spike-evoked fluorescence transients with a greater FWHM than in axon. This difference was not measured in trials when the structures were simultaneously illuminated with large diameter spot "pWF." (g) Similar to (f), CGH-shaped dye excitation revealed faster rise times in axonal versus dendritic spike-evoked fluorescence transients, a difference not observed with "pWF" illumination. Measurements for $(\mathrm{f})$ and $(\mathrm{g})$ were taken from ROls in which CGH excitation and widefield imaging are parfocal with the quantified structure. Asterisks $\left({ }^{*}\right)$ indicate Student's $t$-test $p$-values $\leq 0.05$.

emanating for the dendrite [Fig. 2(b)] and axon [Fig. 2(c)] were collected in separate trials since these structures occupied two different planes of focus. Figure 2(e) redisplays these two traces, amplitude normalized and peak aligned to show the difference in spike kinetics undetectable with pWF illumination [Fig. 2(a)]. Trials interleaved in which neighboring axons and dendrites were simultaneously illuminated with large spots did not show differences [Fig. 2(a), 2(f), and 2(g), $p$-values $>0.3$ Student's $t$ test] in spike kinetics in the same axonal (FWHM: $1.87 \mathrm{~ms} \pm$ 0.09 S.E.M.; rise time: $618.5 \mu$ s \pm 68.2 S.E.M.; $n=4$ trials in two cells) and dendritic ROIs (FWHM: $1.80 \mathrm{~ms} \pm 0.15$ S.E.M.; rise time: $600.2 \mu \mathrm{s} \pm 170.2$ S.E.M.; $n=3$ trials in two cells), even though these structures occupied different planes of focus.

\subsection{Targeted Excitation Increases Fractional Spike-Evoked Transient Amplitude but not Signal-To-Noise}

Paired comparisons of CGH shaped ( $n=4)$ or "pWF" $(n=4)$ trials revealed significantly decreased background fluorescence $\left(F_{\mathrm{Bkg}}=F_{\mathrm{BL}}-F_{\text {dark }}\right.$, averaged over time postshutter opening and prespike), increased RMS noise and increased peak action potential evoked $\mathrm{d} F / F$ [d $F / F_{\text {MAX }}$ summarized by Fig. 3(table)]. Although inhomogeneous partitioning of the lipophyllic voltage dye precludes comparison of the signal amplitude between axonal and dendritic compartments, here we compare $\mathrm{d} F / F_{\mathrm{MAX}}$ emanating from the same ROIs under sculpted and pWF 


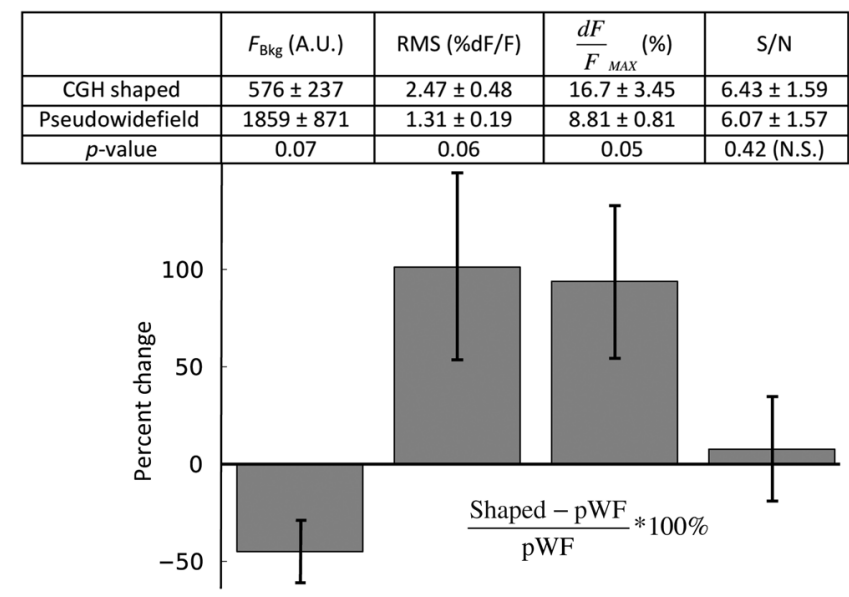

Fig. 3 Comparison of baseline fluorescence, signal noise, spikewaveform amplitude, and signal-to-noise ratio $(\mathrm{S} / \mathrm{N})$. The table shows mean and standard error of the mean for trials taken with CGH shaped VSD excitation and large-spot "pWF" excitation for: (1) background fluorescence $\left(F_{\mathrm{Bkg}}=F_{\mathrm{BL}}-F_{\text {dark }}\right.$, postshutter opening and prespike) fluorescence in arbitrary units (A.U.) after dark (preshutter opening) value subtraction; (2) root-mean-square (RMS) noise of the prespike signal $(\% \mathrm{~d} F / F)$; (3) maximum $\mathrm{d} F / F(\%)$ of the action potentialevoked fluorescence transient, and; (4) the $\mathrm{S} / \mathrm{N}$ ratios. Paired Student's $t$-tests indicate significant increases in RMS noise and peak $\mathrm{d} F / F$, and reduction baseline fluorescence between $\mathrm{CGH}$ shaped and pWF trials, illustrated as a percent change in the bar graph (lower). There was no significant difference in $\mathrm{S} / \mathrm{N}$ between CGH shaped and pWF trials.

illumination conditions. Since both the signal $\left(\mu=\mathrm{d} F / F_{\text {MAX }}\right)$ and RMS noise increased with CGH-shaped excitation, we observed no change in $\mathrm{S} / \mathrm{N}$.

\section{Discussion}

We demonstrate that the CGH light targeting to neuronal structures enables functional fluorescence transient spatial specificity unachievable with widefield epifluorescence. Importantly, differences in action potential kinetics between adjacent axons and dendrites could be discerned with targeted CGH illumination, but not with pWF illumination. This gain in spatial specificity, despite reducing baseline fluorescence and total photon flux, did not decrease signal-to-noise ratios (Fig. 3, S/N). Specifically, the increased baseline noise $\left(\mathrm{RMS}_{\mathrm{BL}}\right)$ was compensated by increased transient $\mathrm{d} F / F$, presumably due to the higher fraction of photons emanating from external membraneembedded fluorophores undergoing synchronous electrochromatic shifts during action potential propagation. In the pWF case, increased baseline fluorescence drives an expected decrease in RMS noise, as shot-noise decreases in proportion to the square root of photon flux. These additional photons could originate from autofluorescence, spilled dye, or labeled structures outside of the focal plane and contribute asynchronously or nonspecifically to the spike-evoked fluorescence transient. Hence, although targeted illumination recruited lower photon fluxes, a higher fraction of light evolved from fluorophores subject to near-simultaneous transmembrane potential changes, as evidenced by increased peak spike-evoked $\mathrm{d} F / F$, on average $16 \%$ compared to $9 \%$ for pWF illumination of the same ROIs (Fig. 3). In certain trials, spike-evoked peak $\mathrm{d} F / F$ approached values closer to theoretically estimated limits for electrochromic probes ${ }^{7,29}$ [for example, 26\% in Fig. 2(b)] than the values typically $(\sim 10 \%$ per $100 \mathrm{mV})$ achieved in axons and dendrites in scattering tissue. ${ }^{2-6}$

In addition to arbitrary lateral patterning capabilities, $\mathrm{CGH}$ light targeting confined fluorescence excitation to $\sim 10 \mu \mathrm{m}$ in the axial direction for light patterns shaped to cover 10 to $20 \mu \mathrm{m}$ segments of axons and dendrites [Fig. 1(c) and 1(d)]. Amplitude-based one-photon methods for lateral light shaping, including light-emitting diode arrays ${ }^{30}$ and digital micromirror devices, ${ }^{31,32}$ do not achieve optical sectioning. Moreover, in contrast with confocal methods for excitation focal volume confinement, CGH enables simultaneous parallel illumination of the entire ROI, removing constraints on the sample rate imposed by serial scanning travel and dwell time. Moreover, CGH provides gains in collection efficiency since fluorescence can be imaged in widefield without losses through pinholes or lens arrays. Increasing spatial specificity without sacrificing information-bearing photons in the collection path is especially important for shot-noise-limited functional fluorescence measurements, in which the $\mathrm{S} / \mathrm{N}$ scales in proportion to the square root of the photon flux. Although not quantified here, the lateral and axial confinement of CGH-shaped excitation may also prevent photodamage in neighboring dye-containing structures. In the future, the axial sectioning capability of CGH can be further exploited in two-photon with temporal focusing, ${ }^{33-35}$ which confines twophoton fluorescence by geometrically dispersing laser pulses, thus reducing peak power outside of the focal plane. Although for the one-photon CGH case axial confinement degrades linearly in proportion to lateral area, ${ }^{26}$ with temporal focusing the lateral area and axial confinement are decoupled for laterally extended regions. ${ }^{35}$ In addition, temporally focused shapes show high robustness to scattering, further recommending this approach for extended shape fluorescence excitation in depth. ${ }^{36,37}$

One undisputable disadvantage of $\mathrm{CGH}$ is the inhomogeneous light distribution within generated light patterns. These intensity "speckles" vary by $15 \%$ to $20 \%$ for one-photon excitation, and up to $\sim 50 \%$ for two-photon, due to the quadratic dependence of the signal on the excitation density. Speckles arise primarily from the approximation in the IFTA and cross talk between adjacent pixels of the LCOS-SLM. Techniques such as rotating diffusers ${ }^{35}$ and phase mask shift-averaging ${ }^{38}$ can average over speckles and smooth the spatial distribution, but at the cost of light efficiency, axial or temporal resolution. Speckle-free illumination can also be accomplished with generalized phase contrast, ${ }^{33,39}$ an interferometric method for light shaping. Overcoming the problem of speckle, especially in the two-photon case, will be important since large intensity fluctuations can exceed photodamage thresholds.

In conjunction with the rapidly expanding toolbox of organic ${ }^{29,40-47}$ and protein-based, genetically targetable voltage, ${ }^{48-57}$ calcium, and neurotransmitter reporters, holographic light shaping can enable high $\mathrm{S} / \mathrm{N}$, parallel detection of neural-evoked fluorescence transients from neighboring cells or subcellular compartments. Here, we have demonstrated that light targeting increases spatial specificity even for cases in which the dye itself is confined to the membrane of a single cell. Targeted light spatial specificity gains could prove more critical with dye bulk loading or with dense expression of genetically targeted voltage-sensitive fluorescent proteins.

\section{Acknowledgments}

This work was supported by the Centre National de la Recherche Scientifique (V. E.), the Agence Nationale de la 
Recherche (grant ANR-10-INSB-04-01, France-BioImaging Infrastructure Network), and a United States National Science Foundation International Research Fellowship (A. J. F). We also thank the FRC and the Rotary Club for financial support through the program "Espoir en Tête" 2012. We thank Leslie Leow (UConn) for providing JPW3028, and Marco Canepari (Grenoble Institut des Neurosciences, France) for lending the Andor 860 EMCCD. We also thank Vincent de Sars for the continued development of "Wavefront Designer" software, and Marc Guillon and Oscar Hernandez for holographic wavefront propagation simulations.

\section{References}

1. M. Popovic, X. Gao, and D. Zecevic, "Voltage-sensitive dye recording from axons, dendrites and dendritic spines of individual neurons in brain slices," J. Visualized Exp. 69, e4261 (2012).

2. S. Antic, G. Major, and D. Zecevic, "Fast optical recordings of membrane potential changes from dendrites of pyramidal neurons," J. Neurophysiol. 82(3), 1615-1621 (1999).

3. A. Foust et al., "Action potentials initiate in the axon initial segment and propagate through axon collaterals reliably in cerebellar Purkinje neurons," J. Neurosci. 30(20), 6891-6902 (2010).

4. A. J. Foust et al., "Somatic membrane potential and Kv1 channels control spike repolarization in cortical axon collaterals and presynaptic boutons," J. Neurosci. 31(43), 15490-15498 (2011).

5. M. A. Popovic et al., "The spatio-temporal characteristics of action potential initiation in layer 5 pyramidal neurons: a voltage imaging study," J. Physiol. 589(Pt 17), 4167-4187 (2011).

6. A. E. Casale and D. A. McCormick, "Active action potential propagation but not initiation in thalamic interneuron dendrites," J. Neurosci. 31(50), 18289-18302 (2011).

7. B. Kuhn, P. Fromherz, and W. Denk, "High sensitivity of Stark-shift voltage-sensing dyes by one- or two-photon excitation near the red spectral edge," Biophys. J. 87(1), 631-639 (2004).

8. J. N. Kerr and W. Denk, "Imaging in vivo: watching the brain in action," Nat. Rev. Neurosci. 9(3), 195-205 (2008).

9. D. A. Dombeck et al., "Optical recording of fast neuronal membrane potential transients in acute mammalian brain slices by second-harmonic generation microscopy," J. Neurophysiol. 94(5), 3628-3636 (2005).

10. L. M. Palmer and G. J. Stuart, "Membrane potential changes in dendritic spines during action potentials and synaptic input," J. Neurosci. 29(21), 6897-6903 (2009).

11. T. Tominaga and Y. Tominaga, "A new nonscanning confocal microscopy module for functional voltage-sensitive dye and $\mathrm{Ca}^{2+}$ imaging of neuronal circuit activity," J. Neurophysiol. 110(2), 553-561 (2013).

12. A. E. Fink et al., "Two-photon compatibility and single-voxel, singletrial detection of subthreshold neuronal activity by a two-component optical voltage sensor," PLoS One 7(8), e41434 (2012).

13. J. A. Fisher et al., "Two-photon excitation of potentiometric probes enables optical recording of action potentials from mammalian nerve terminals in situ," J. Neurophysiol. 99(3), 1545-1553 (2008).

14. C. D. Acker, P. Yan, and L. M. Loew, "Single-voxel recording of voltage transients in dendritic spines," Biophys. J. 101(2), L11-L13 (2011).

15. J. E. Curtis, B. A. Koss, and D. G. Grier, "Dynamic holographic optical tweezers," Opt. Commun. 207, 169 (2002).

16. M. Canepari, M. Djurisic, and D. Zecevic, "Dendritic signals from rat hippocampal CA1 pyramidal neurons during coincident pre- and postsynaptic activity: a combined voltage- and calcium-imaging study," J. Physiol. 580(Pt. 2), 463-484 (2007).

17. M. Polin et al., "Optimized holographic optical traps," Opt. Express 13(15), 5831-5845 (2005).

18. M. Zahid et al., "Holographic photolysis for multiple cell stimulation in mouse hippocampal slices," PLoS One 5(2), e9431 (2010).

19. R. W. Gerchberg and W. O. Saxton, "A pratical algorithm for the determination of the phase from image and diffraction pictures," Optik 35, 237-246 (1972)

20. J. R. Fienup, "Phase retrieval algorithms: a comparison," Appl. Opt. 21, 2758-2769 (1982).
21. F. Wyrowski and O. Bryngdahl, "Iterative Fourier-transform algorithm applied to computer holography," J. Opt. Soc. Am A 5, 1058 (1988).

22. Z. Zalevsky, D. Mendlovic, and R. G. Dorsch, "Gerchberg-Saxton algorithm applied in the fractional Fourier or the Fresnel domain," Opt. Lett. 21, 842-844 (1996).

23. M. Frigo and S. G. Johnson, "The design and implementation of FFTW3," Proc. IEEE 93(2), 216-231 (2005).

24. A. Edelstein et al., Computer control of microscopes using $\mu$ manager," Curr. Protoc. Mol. Biol. 14.20.1-14.20.17 (2010).

25. W. S. Rasband, "ImageJ," U. S. National Institutes of Health, Bethesda, Maryland (1997-2014) http://imagej.nih.gov/ij/.

26. C. Lutz et al., "Holographic photolysis of caged neurotransmitters," Nat. Methods 5(9), 821-827 (2008).

27. G. Stuart et al., "Action potential initiation and backpropagation in neurons of the mammalian CNS," Trends Neurosci. 20(3), 125-131 (1997).

28. Y. Shu et al., "Modulation of intracortical synaptic potentials by presynaptic somatic membrane potential," Nature 441(7094), 761-765 (2006).

29. G. Hubener, A. Lambacher, and P. Fromherz, "Anellated hemicyanine dyes with large symmetrical solvatochromism of absorption and fluorescence," J. Phys. Chem. B 107(31), 7896-7902 (2003).

30. N. Grossman et al., "Multi-site optical excitation using ChR2 and micro-LED array," J. Neural Eng. 7(1), 16004 (2010).

31. L. J. Horneck, " 128 X 128 deformable mirror device," IEEE Trans. Electron. Devices 30(5), 539-545 (1983).

32. C. W. Liang et al., "Patterned photostimulation with digital micromirror devices to investigate dendritic integration across branch points," J. Visualized Exp. 49, 2003 (2011).

33. E. Papagiakoumou et al., "Scanless two-photon excitation of channelrhodopsin-2," Nat. Methods 7(10), 848-854 (2010).

34. E. Papagiakoumou et al., "Temporal focusing with spatially modulated excitation," Opt. Express 17, 5391-5401 (2009).

35. E. Papagiakoumou et al., "Patterned two-photon illumination by spatiotemporal shaping of ultrashort pulses," Opt. Express 16, 22039-22047 (2008).

36. E. Papagiakoumou et al., "Functional patterned multiphoton excitation deep inside scattering tissue," Nat. Photonics 7(4), 274-278 (2013).

37. A. Begue et al., "Two-photon excitation in scattering media by spatiotemporally shaped beams and their application in optogenetic stimulation," Biomed. Opt. Express 4(12), 2869-2879 (2013).

38. L. Golan and S. Shoham, "Speckle elimination using shift-averaging in high-rate holographic projection," Opt. Express 17(3), 1330-1339 (2009).

39. J. Glückstad, "Phase contrast image synthesis," Opt. Commun. 130, 225 (1996).

40. E. W. Miller et al., "Optically monitoring voltage in neurons by photoinduced electron transfer through molecular wires," Proc. Natl. Acad. Sci. U. S. A. 109(6), 2114-2119 (2012).

41. L. B. Cohen, B. M. Salzberg, and A. Grinvald, "Optical methods for monitoring neuron activity," Ann. Rev. Neurosci. 1, 171-182 (1978).

42. A. Grinvald et al., "Optical imaging of neuronal activity," Physiol. Rev. 68(4), 1285-1366 (1988).

43. A. Grinvald and R. Hildesheim, "VSDI: a new era in functional imaging of cortical dynamics," Nat. Rev. Neurosci. 5(11), 874-885 (2004).

44. D. S. Peterka, H. Takahashi, and R. Yuste, "Imaging voltage in neurons," Neuron 69(1), 9-21 (2011).

45. P. Fromherz et al., "ANNINE-6plus, a voltage-sensitive dye with good solubility, strong membrane binding and high sensitivity," Eur. Biophys. J.: EBJ 37(4), 509-514 (2008).

46. A. L. Obaid et al., "Novel naphthylstyryl-pyridium potentiometric dyes offer advantages for neural network analysis," J. Neurosci. Methods 134(2), 179-190 (2004).

47. J. P. Wuskell et al., "Synthesis, spectra, delivery and potentiometric responses of new styryl dyes with extended spectral ranges," J. Neurosci. Methods 151(2), 200-215 (2006).

48. P. Zou et al., "Bright and fast multicoloured voltage reporters via electrochromic FRET," Nat. Commun. 5, 4625 (2014).

49. W. Akemann et al., "Imaging neural circuit dynamics with a voltage-sensitive fluorescent protein," J. Neurophysiol. 108(8), 2323-2337 (2012).

50. W. Akemann et al., "Imaging brain electric signals with genetically targeted voltage-sensitive fluorescent proteins," Nat. Methods 7(8), 643-649 (2010). 
51. B. J. Baker et al., "Genetically encoded fluorescent voltage sensors using the voltage-sensing domain of Nematostella and Danio phosphatases exhibit fast kinetics," J. Neurosci. Methods 208(2), 190-196 (2012).

52. L. Barnett et al., "A fluorescent, genetically-encoded voltage probe capable of resolving action potentials," PLoS One 7(9), e43454 (2012).

53. J. M. Kralj et al., "Optical recording of action potentials in mammalian neurons using a microbial rhodopsin," Nat. Methods 9(1), 90-95 (2012).

54. J. M. Kralj et al., "Electrical spiking in Escherichia coli probed with a fluorescent voltage-indicating protein," Science 333(6040), 345-348 (2011).

55. Y. Gong, J. Z. Li, and M. J. Schnitzer, "Enhanced archaerhodopsin fluorescent protein voltage indicators," PLoS One 8(6), e66959 (2013).

56. F. St-Pierre et al., "High-fidelity optical reporting of neuronal electrical activity with an ultrafast fluorescent voltage sensor," Nat. Neurosci. 17(6), 884-889 (2014).

57. L. Jin et al., "Single action potentials and subthreshold electrical events imaged in neurons with a fluorescent protein voltage probe," Neuron 75(5), 779-785 (2012).

Amanda J. Foust is a National Science Foundation international postdoctoral fellow in the Department of Neurophotonics at Paris Descartes University, adapting and optimizing computer-generated holography methods for brain circuit reverse engineering. Amanda began optical neurophysiology research in the laboratory of David Rector at Washington State University (Pullman, Washington; BS 2006). She holds MPhil and PhD degrees from Yale University where she used voltage-sensitive dyes to study axonal information processing in the laboratory of David McCormick.

Valeria Zampini is a research engineer at Paris Descartes University, Paris, France, specializing in electrophysiology. She previously worked as a postdoc at the École normale supérieure, Paris,
France (2010 to 2013), where she studied synaptic integration in unipolar brush cells interneurons of the vestibulo-cerebellum. During her $\mathrm{PhD}$ and first postdoctoral training she studied vestibular and cochlear hair cells physiology and $\mathrm{Ca}_{\mathrm{v}} 1.3$ calcium channels responsible for synaptic release in these receptors (University of Pavia, Italy; University of Sheffield, United Kingdom).

Dimitrii Tanese received his BA and MS degrees from the University of Bologna, Italy. He obtained his $\mathrm{PhD}$ degree from the University Pierre et Marie Curie in Paris, working on semiconductor microcavities. $\mathrm{He}$ is a postdoctoral fellow from 2013 until the present at the Neurophotonics Laboratory in Paris. He is currently involved in the development of optical methods for voltage imaging and neuronal photoactivation based on shaped illumination.

Eirini Papagiakoumou is a researcher at the Neurophotonics Laboratory of Paris Descartes University. She obtained her PhD degree in physics at the National Technical University of Athens working on cell micromanipulation with optical tweezers, laser ablation of tissues and waveguiding laser radiation. Her current interests focus on the use of wavefront engineering techniques and nonlinear phenomena on two-photon microscopy, applied in neuroscience and principally in high resolution neuronal photoactivation and imaging.

Valentina Emiliani is the director of the Neurophotonics Laboratory. She obtained her PhD degree in physics at the University 'La Sapienza' Rome. As a postdoc, she investigated carrier transport in quantum wires (Max Born Institut, Berlin) and light propagation in disordered structures (LENS, Florence) by SNOM and cell mechanotransduction by optical tweezers (Institute Jacques Monod, Paris). In 2005, she formed the "wavefront engineering microscopy" group at Paris Descartes University, pioneering the use of wavefront shaping for neuroscience. 